\title{
Technology Research on Information Analysis of Internet + Ecological Factors of Ginseng
}

\author{
Hongwei Zhu
}

Jilin Agricultural Science and Technology University, Jilin, 132000, China

Keywords: Information analysis, Ecological factors, Ginseng

\begin{abstract}
Ginseng ecological factor is a variety of environmental factors that have influence on ginseng. It has a direct effect on ginseng, which mainly affects the survival, reproduction, distribution, function and structure of ginseng. Internet plus ginseng ecological factor analysis of information technology includes information acquisition technology, information tracing technology and information visualization technology. This paper gives a detailed analysis of the applications of these technologies to provide some references for information technology research of the internet plus ginseng ecological factor.
\end{abstract}

\section{Introduction}

Ginseng has a fleshy root, medicinal, saponins, volatile oil, containing a variety of organic acids, polysaccharides, vitamins and amino acids, choline and other chemical components, the main effective components of ginseng are ginsenosides content in different organs in different terpenoid biosynthesis structural unit in the form of ginsenoside. Terpenoid glycosides, namely ginsenosides, have many kinds of medicinal functions, such as anti-inflammatory, anti-tumor, anti-oxidation, inhibiting cell apoptosis, and so on. Ginseng ginsenosides are relatively low in ginseng plants. Therefore, based on the analysis of ginsenoside biosynthesis, structure and function of the use of biological information, in-depth study of the key enzyme genes of methodology, in order to make use of biological techniques to regulate these genes of key enzymes activity, to improve the content of saponins in ginseng, ten points will be beneficial. Ginseng has an important position in the treasure house of Chinese medicine, and enjoys the reputation of the king of hundred grass and the first of the hundred drugs. Chinese ginseng is mainly Jilin ginseng, although the development of ginseng industry has begun to take shape, the status of industry is not optimistic. China's ginseng production accounts for $70 \%$ of the world's total. In addition, nearly 2 enterprises in China are engaged in planting, processing and trading of ginseng. Small and scattered brands are missing, leading to many enterprises that can only rely on foreign enterprises or strong brands. The above statistics show that China's ginseng industry is facing a big country with a large output and a small industry. One reason is the technology of ginseng cultivation, production, processing and other aspects of backward, aging equipment, ginseng related food quality cannot adapt to the market development needs; the other side is the market for a long time in ginseng planting and harvesting, processing, circulation and other business aspects of the lack of a unified and standardized management, which seriously affected the quality of ginseng products and market competitiveness.

\section{Information Acquisition Technology of Internet + Ecological Factors of Ginseng}

The core function of the mobile terminal data collection includes the spatial, attribute and time information collection of ginseng ecological factors. This system uses the more mature ArcGIS for Android/IOS development package of ESRI company, and provides a rich map loading and editing API. The most flow - consuming map can be downloaded in the WIFI environment or prepositioned into the mobile terminal, which can reduce the flow of 90\%. Making offline map using ArcGIS10.1, the map after registration in accordance with the Pyramid section structure package, provided to the 
mobile terminal to download on the server TPK file, the mobile terminal to instantiate ArcGISLocalTiledLayer layer, and added to the displayed map container in the Map View class. Information collection is generally a field survey. In order to get the current position, that is, to make the map center point as the current latitude and longitude coordinates, the GPS module of the mobile phone needs to be called. (in the case of Android) first need to use Location Manager (isProisProviderEnabled) to determine the GPS satellite positioning or WLAN/3G/2G assisted GPS is available, if not available through the Intent (Settings. ACTION_LOCATION_SOURCE_ SETTINGS) jump to control page convenient for users to open the GPS function. By implementing the on Location Changed (Location location) method in the Location Listener interface, we get the latitude and longitude coordinates of location and encapsulate it into Point class. Finally, we map the map to the current location by MapView.centerAt (Point centerPt, Boolean animated). The positioning accuracy of smart phone in the field is generally 15 to $30 \mathrm{~m}$. Users can identify and confirm the accurate location of the collection point (the current point or the polygon vertex of the pest area) through offline map comparison, and the location accuracy is up to $15 \mathrm{~m}$. The occurrence of ginseng ecological factors may be a few plants, that is, point elements, or a region, that is, the surface elements. Based on map loading, users just click on the map to achieve point and surface elements editing and automatic rendering. The background can capture the geographic location of user clicks by implementing the onSingleTap (MotionEvente) method, in which the surface elements are the collection of vertices of polygons, which are stored in the ArrayList $<$ Point $>$ set. When the information is collected, the user information can be easily read, and it is not necessary to refill it again, and the operation is simplified. The realization of ginseng ecological factors and species collecting keys the user clicks on the navigation bar to enter the selection interface, select the ginseng ecological factors and species to be collected, automatically determine the positioning function is turned on, if not jump to the setup, ginseng ecological factor information collection page opens after entering the automatic loading map system, and map display according to a certain proportion to the current point according to the read position. If the single area can choose the above point collection, click the location of ginseng ecological factor directly on the map, if the large area can choose the surface to collect, click on the map to click the location of the location of the area, and the system automatically completes rendering and rendering. Users can click the level selection button according to ginseng ecological factor grading standard, select ginseng ecological factor level, and click date to modify ginseng ecological factor acquisition time. After the information collection is completed, the information can be sent to the server by clicking on the submission of the upper right corner.

\section{Information Traceability Technology of Internet + Ecological Factors of Ginseng}

\subsection{Information Traceability Technology in Processing}

Traceability Technology is a process to restore and control the whole process and historical trajectory of products, and the ability to generate places and sales channels, to achieve traceability and traceability of product information trajectory. Traceability is the ability to trace the history, application, and place of an entity based on the identification of a product. The establishment, improvement and improvement of traceability system for agricultural products is effective in dealing with frequent food safety problems. From 2007 to 2009, 3 central No.1 documents set clear requirements for the establishment of traceability system for agricultural products quality and safety. As a single identity, traceability source is used to identify monomer identification information and monomer record data in the library, and it is the key to read, read, trace and source information for the traceability system. According to the characteristics of ginseng and its products in different links, this system adopts different information carrier identification types, and designs content representation rules of different types of information carriers, respectively. RFID label information is large and easy to read and write, so it is used in the processing and storage of ginseng. According to the requirement of data element in processing and storage links, the coding principle of EPC-SGTIN-96 electronic label is explained by hierarchical classification method. First (8): header EPC-SCTIN-96 header 
encoding for 00110000, only second: determine the label type; filter value (3 bits), used to identify the type of raw materials, encoding followed by 000 to 111, said the common primary categories of raw materials to be identified as 010 ; third: value ( 3 a 82 bit encoding), marking the next raw ginseng products identification and identification of the specific bit allocation scheme for the setting, can be more flexible for a given bit allocation, in order to adapt to the ginseng product processing process RFID code changes. This system uses the corresponding bit allocation scheme, which is identified as bit processors, ginseng products identified. Level fourth: raw material reference mark, which is used to identify the reference material for a given raw material. Level fifth: the ginseng product logo, which is the only identifier for the finished product.

\subsection{Information Traceability Technology in Planting}

QR codes with fast decoding, data density, space and without vertical linear scanning characteristics, therefore for the ends of ginseng traceability links, namely ginseng planting links and ginseng sales information traceability, specifically for the individual identification of raw materials packaging box and ginseng ginseng products monomer. Because of the same planting environment, ginseng has the same batch number, and raw ginseng according to its grade is different packaging specifications. Therefore, monomer packaging is generally ranging from dozens to hundreds of branches. The single package information of the ginseng is directly attached to the outside of the packing box by the QR code. The quality and safety traceability system of ginseng is designed as a unified access module, a basic processing module and a data processing module from the overall framework of the functional angle. The uniform access module provides users access management, multi class user login management, single point form display, basic information query and release management; basic processing module mainly includes the basic data acquisition and adjustment, form management and traceability code management, transaction management and other basic external equipment platform docking interface and data interaction; the processing module includes data storage and backup, disaster recovery, the basic functions of data analysis and verification, data query, early warning and advanced safety management and certification. The system uses the B/S mode, and the specific functions are realized through the unified access platform, the basic application platform and the data warehouse platform. The unified access platform is responsible for interaction with users, providing multi-user single point access, report display and friendly interface designed by Web Dynpro, and the basic application platform is responsible for business logic implementation. The data warehouse platform realizes the advanced query and report function of the data.

\subsection{Information Traceability Technology in Marketing}

The information collection of ginseng sales compared to the raw materials of planting ginseng and ginseng products link processing, sales due to various retail distributors, information collection points are more dispersed, so bring difficulty for the information source, any distribution of missing information will affect the integrity of the whole traceability chain. Therefore, to solve this problem, the traceability system in the design process in order to facilitate traceability links in the regulatory departments, consumers and enterprises for planting and processing information, special access records on the basis of sales information, to design a QR code identification as the carrier of the circulation of electronic form, according to the sales data link specifications were designed for consumers for the right information at all levels and retail distributors were recorded for traceability information goods. The consumer query code detailed information for consumers to obtain goods through mobile phone barcode, circulation of electronic form is used to record information tracking records of goods at all levels of distributors and sub distributors in the circulation process, detailed records through electronic form can clearly show the circulation of commodities. The design of electronic form is a good solution to the problem of the lack of traceability information because of the complexity of the distribution and circulation of ginseng products. The design of the circulation electronic form is also applicable to the standard management of the traceability and circulation of other products. The system designs and implements information covering planting and harvesting, 
including planting environment information, production material input and so on. Production and processing links include processing basic information, processing technology and information, and operation and circulation links, including commodity orientation and basic information of distributors at all levels. This system in the design and implementation of quality and safety of ginseng products covering planting and harvesting processing and operation of production, circulation of the whole industry chain information traceability system process, aim to solve the following key problems: regulate traceability links key points data; according to the particularity of labeling and tracing key monomer information, design and implementation of RFID and two dimensional bar code based on identification of ginseng and ginseng products; the use of integrated development platform technology to achieve traceability system.

\section{Information Visualization Technology of Internet + Ecological Factors of Ginseng}

The system adopts ArcGISCatalog10.1 Oracle database using the Oracle client to read, because Oracle data is written into spatial data, using ArcMap software can be directly loaded in the form of pest layer and the information, and use the QueryLayer function calls the SQL statement to insect category and time setting conditions of screening specific information for further operations. The distribution of pests and diseases in cotton field is characterized by spatial aggregation. The system adopts the inverse distance weighting method based on similarity principle to predict the occurrence of insect pests near the point in the collected area. It is assumed that each known sample point has a local influence on the prediction of the prediction point, and its influence decreases with the decrease of the distance. Based on the analysis of the structure characteristics of ginseng HMGR gene encoding protein, further on the function of the GO KEGG InterPro pathway annotation classification were prospectively predicted from the biological process, cellular component and molecular function three aspects were expounded the function of PgHMGR gene; and HMGR enzyme with oxidoreductase activity HMG two acyl coenzyme A reductase activity and NADP binding ability of coenzyme binding capacity. However, the bioinformatics analysis of PgHMGR gene sequence carried out by our institute is only through computer software analysis. Its gene function and biological function of protein need further research and verification. PgHMGR gene is a ginsenoside biosynthetic pathway in a key enzyme gene in ginsenoside biosynthesis and many related enzyme genes, in future research, bio information analysis system for all genes of key enzymes in the pathway, is the key enzyme for further genetic transformation and the high expression of foundation at the same time, ginseng ginseng genome research resources system analysis, ginseng breeding, gene mapping and cloning and natural population protection to provide the necessary information resources. Ginsenoside is an important part of ginseng secondary metabolites. Its content and composition mainly depend on the key enzymes of biosynthesis. These compounds have high economic value. Progress has been made in the study of ginsenoside biosynthesis. However, because its synthetic pathway is a dynamic process and regulated by many factors, there are still some difficulties in the research. The first step in the study of ginsenoside biosynthesis pathway is to obtain the basic biological information of the genes and related genes of all the key enzymes in the whole pathway.

\section{Conclusion}

Ginseng products quality and safety traceability system and visualization system combined with key point data specification technology, RFID and barcode logo design and integrated R \& D platform technology. Technology includes coverage of planting and harvesting, including planting environment information, production material input and other information. Production and processing links include processing basic information, processing technology information, and operation and circulation links, including commodity orientation and basic information of distributors at all levels. 


\section{Acknowledgement}

The paper is the result of the Science and Technology Development Plan Project of Natural Science Foundation of Jilin Province named "Research on Monitoring Technology Environmental Parameters of Ginseng Growth Using Wireless Sensor Network” (Grant No. 20150101099JC) led by Hongwei Zhu, Construction Project of Technology Innovation Center Base and Service Platform of Development Plan of Science and Technology Department of Jilin Province named "Analysis and Prediction of Information Service Platform of Internet + Ecological Factors of Ginseng” led by Laiwu Yi and Hongwei Zhu and Key Subject Cultivation Project of Jilin Agricultural Science and Technology University named "Research on Monitoring System of Ginseng Growth Environment Parameters Based on IoT Technology" (Grant No. X097 of Jilin Agricultural Science and Technology University in 2015) led by Hongwei Zhu.

\section{References}

[1] Jia Guanglin, Huang Liufang, Suo Fengmei, et al. Correlation between ginsenoside contents in Panax ginseng roots and ecological factors, and ecological division of ginseng plantation in China [J]. Chinese Journal of Plant Ecology, 2012, 36 (4): 302-312.

[2] Xie Caixiang, Suo Fengmei, Jia Guanglin, et al. Correlation between ecological factors and ginsenosides [J]. Acta Ecologica Sinica, 2011, 31(24): 7551-7563.

[3] He Wenbin, Zhang Junlong, Chen Naihong. Protein kinase-based neural signaling pathways for ginsenosides: a retrospective review [J]. Journal of Traditional Chinese Medicine, 2015, 35(6): 349-354.

[4] Zhang Yuefei, Li Yali, Liu Zhe, et al. Dynamic Microwave-Assisted Extraction of Total Ginsenosides from Ginseng Fibrous Roots [J]. Wuhan University Journal of Na tural Sciences, 2015, 20(3): 247-254. 